\title{
DIII topological superconductivity with emergent time-reversal symmetry
}

\author{
Christopher Reeg, ${ }^{1}$ Constantin Schrade, ${ }^{1,2}$ Jelena Klinovaja, ${ }^{1}$ and Daniel Loss ${ }^{1}$ \\ ${ }^{1}$ Department of Physics, University of Basel, Klingelbergstrasse 82, CH-4056 Basel, Switzerland \\ ${ }^{2}$ Department of Physics, Massachusetts Institute of Technology, Cambridge, Massachusetts 02139, USA
}

(Received 23 August 2017; published 25 October 2017)

\begin{abstract}
We find a class of topological superconductors which possess an emergent time-reversal symmetry that is present only after projecting to an effective low-dimensional model. We show that a topological phase in symmetry class DIII can be realized in a noninteracting system coupled to an $s$-wave superconductor only if the physical time-reversal symmetry of the system is broken, and we provide three general criteria that must be satisfied in order to have such a phase. We also provide an explicit model which realizes the class DIII topological superconductor in 1D. We show that, just as in time-reversal invariant topological superconductors, the topological phase is characterized by a Kramers pair of Majorana fermions that are protected by the emergent time-reversal symmetry.
\end{abstract}

DOI: 10.1103/PhysRevB.96.161407

Introduction. Topological superconductors have been intensively pursued in recent years [1-3] because the Majorana fermions which are localized to their boundaries have potential applications in the development of a topological quantum computer $[4,5]$. The most promising proposals to date for engineering topological superconductivity involve coupling a conventional superconductor either to a nanowire with Rashba spin-orbit interaction that is subjected to an external magnetic field [6-26] or to a ferromagnetic atomic chain [27-34].

Additionally, there have been several proposals to engineer topological superconductors in symmetry class DIII. Such systems possess both particle-hole symmetry and time-reversal symmetry [35], with the presence of time-reversal symmetry ensuring that the Majorana fermions existing at the boundaries of class DIII topological superconductors come in Kramers pairs. In one dimension (1D), where superconductivity is required to be induced by the proximity effect, it has been shown that a nontrivial topological phase in class DIII can be realized by proximity coupling a noninteracting multichannel Rashba nanowire to an unconventional superconductor [36-39] or to two conventional superconductors forming a Josephson junction with a phase difference of $\pi$ [40]. Alternatively, an effective $\pi$-phase difference can be induced in a multichannel Rashba nanowire with repulsive electron-electron interactions [41] or in a system of two topological insulators coupled to a conventional superconductor via a magnetic insulator [42]. It has also been proposed to realize class DIII topological superconductivity in a system of two Rashba nanowires [43-45] or two topological insulators [46] coupled to a single conventional superconductor, but repulsive interactions are also necessary to reach the topological phase in these setups, which require a strength of induced crossed Andreev (interwire) pairing exceeding that of the direct (intrawire) pairing [47-49]. While it would be beneficial to engineer a DIII topological superconductor in a noninteracting 1D system coupled to a single conventional superconductor, as such a setup could avoid relying on unconventional superconductivity or interactions that are difficult to control experimentally, it has been shown that this is not possible in a fully time-reversal invariant system $[38,50]$.

In this paper, we show that such a $1 \mathrm{D}$ topological superconductor in class DIII can be realized when time-reversal symmetry is explicitly broken. While the full Hamiltonian (describing the 1D system, the superconductor, and the tunnel coupling) possesses only particle-hole symmetry and is thus in symmetry class D, it is possible to place the system in symmetry class DIII after integrating out the superconductor [47,51-57] and projecting to an effective 1D model. (This is completely analogous to the case of a single Rashba nanowire coupled to an $s$-wave superconductor and subjected to an external magnetic field [6,7]. In this setup, it is possible to define an effective time-reversal symmetry, which squares to +1 , and to place the system in class BDI after projecting to $1 \mathrm{D}$ [58].) We establish three necessary criteria to realize a DIII topological phase. First, the 1D system must obey an "emergent" time-reversal symmetry. That is, given the Hamiltonian density $h_{k}$ of the 1D system, there must exist a unitary matrix $\mathcal{T}_{1 \mathrm{D}}$ such that $\mathcal{T}_{1 \mathrm{D}}^{\dagger} h_{k} \mathcal{T}_{1 \mathrm{D}}=h_{-k}^{*}$ and $\mathcal{T}_{1 \mathrm{D}}^{2}=-1$. [While a specific example could be the physical time-reversal symmetry $\mathcal{T}_{1 \mathrm{D}}=i \sigma_{y}$, where $\sigma_{x, y, z}$ is a Pauli matrix acting in spin space, we do not restrict ourselves to this case.] Second, the self-energy induced on the 1D system by the superconductor must preserve the emergent time-reversal symmetry. Third, the anomalous (pairing) component of the self-energy must have both positive and negative eigenvalues.

After a general discussion, we provide a simple model which realizes the DIII topological phase in 1D. We consider a system of two Rashba nanowires with opposite Zeeman splittings coupled to an $s$-wave superconductor. We show that such a system undergoes a topological phase transition under certain conditions. By explicitly solving for the wave functions of the Majorana bound states, we show that the topological phase is characterized by the presence of a Kramers pair of Majorana fermions that is protected by the emergent time-reversal symmetry.

Minimum requirements for DIII topological phase. We consider a general 1D (noninteracting) system coupled to a conventional superconductor. We assume that the system is translationally invariant along the direction of the $1 \mathrm{D}$ system, allowing us to define a conserved momentum $k$. The Hamiltonian of the 1D system is given by

$$
H_{1 \mathrm{D}}=\int \frac{d k}{2 \pi} \psi_{k}^{\dagger} h_{k} \psi_{k},
$$


where $\psi_{k}$ is a spinor annihilation operator acting on all internal degrees of freedom of the 1D system (spin, subband, etc.) and $h_{k}$ is a Hermitian matrix. We assume that there exists a unitary matrix $\mathcal{T}_{1 \mathrm{D}}$ which acts as an effective time-reversal symmetry on the Hamiltonian, such that $\mathcal{T}_{1 \mathrm{D}}^{\dagger} h_{k} \mathcal{T}_{1 \mathrm{D}}=h_{-k}^{*}$ and $\mathcal{T}_{1 \mathrm{D}}^{2}=-1$. Introducing the Nambu spinor $\Psi_{k}^{\dagger}=\left(\psi_{k}^{\dagger}, \psi_{-k}^{T} \mathcal{T}_{1 \mathrm{D}}\right)$, the Hamiltonian Eq. (1) can be expressed as

$$
H_{1 \mathrm{D}}=\frac{1}{2} \int \frac{d k}{2 \pi} \Psi_{k}^{\dagger} \mathcal{H}_{k}^{1 \mathrm{D}} \Psi_{k},
$$

where $\mathcal{H}_{k}=\tau_{z} h_{k}$ and $\tau_{x, y, z}$ is a Pauli matrix acting in Nambu space.

The 1D system is coupled to a conventional superconductor which is described by a BCS Hamiltonian,

$$
H_{\mathrm{sc}}=\frac{1}{2} \int \frac{d k}{2 \pi} \int d \mathbf{r}_{\perp} \eta_{k}^{\dagger}\left(\mathbf{r}_{\perp}\right) \mathcal{H}_{k}^{\mathrm{sc}}\left(\mathbf{r}_{\perp}\right) \eta_{k}\left(\mathbf{r}_{\perp}\right),
$$

where $\mathbf{r}_{\perp}$ denotes directions transverse to the 1D system and $\eta_{k}^{\dagger}=\left(\eta_{k \uparrow}^{\dagger}, \eta_{k \downarrow}^{\dagger},-\eta_{-k \downarrow}, \eta_{-k \uparrow}\right)$ is a spinor creation operator in Nambu $\otimes$ spin space. The Hamiltonian density in this basis is given by $\mathcal{H}_{k}^{\mathrm{sc}}\left(\mathbf{r}_{\perp}\right)=\tau_{z}\left[\left(k^{2}-\nabla_{\perp}^{2}\right) / 2 m_{s}-\mu_{s}\right]+\tau_{x} \Delta$, where $m_{s}, \mu_{s}$, and $\Delta$ are the effective mass, chemical potential, and pairing potential of the superconductor, respectively. The superconductor is time-reversal invariant, $\mathcal{T}_{\mathrm{sc}}^{\dagger} \mathcal{H}_{k}^{\mathrm{sc}} \mathcal{T}_{\mathrm{sc}}=\mathcal{H}_{-k}^{\mathrm{sc} *}$, where $\mathcal{T}_{\text {sc }}=i \sigma_{y}$ is the physical time-reversal operator, with $\sigma_{x, y, z}$ the Pauli matrix acting in spin space.

We allow for a linear coupling between the 1D system and the superconductor of the form

$$
H_{t}=\frac{1}{2} \int \frac{d k}{2 \pi} \int d \mathbf{r}_{\perp}\left[\eta_{k}^{\dagger}\left(\mathbf{r}_{\perp}\right) T_{k}\left(\mathbf{r}_{\perp}\right) \Psi_{k}+\text { H.c. }\right] .
$$

The tunneling matrix in Nambu space, $T_{k}\left(\mathbf{r}_{\perp}\right)$, can be expressed generally as

$$
T_{k}=T_{k}^{0}+\tau_{z} T_{k}^{z}
$$

where $T_{k}^{0}=\left[t_{k}-\mathcal{T}_{\mathrm{sc}}^{\dagger} t_{-k}^{*} \mathcal{T}_{1 \mathrm{D}}\right] / 2, T_{k}^{z}=\left[t_{k}+\mathcal{T}_{\mathrm{sc}}^{\dagger} t_{-k}^{*} \mathcal{T}_{1 \mathrm{D}}\right] / 2$, and $t_{k}$ is a tunneling matrix acting on all additional degrees of freedom in the system [59]. Combining Eqs. (2)-(4), the full Hamiltonian can be expressed as

$$
H=\frac{1}{2} \int \frac{d k}{2 \pi}\left(\begin{array}{ll}
\Psi_{k}^{\dagger} & \eta_{k}^{\dagger}
\end{array}\right)\left(\begin{array}{cc}
\mathcal{H}_{k}^{1 \mathrm{D}} & T_{k}^{\dagger} \\
T_{k} & \mathcal{H}_{k}^{\mathrm{sc}}
\end{array}\right)\left(\begin{array}{c}
\Psi_{k} \\
\eta_{k}
\end{array}\right),
$$

where we suppress explicit reference to $\mathbf{r}_{\perp}$ for brevity. Note that the time-reversal symmetry of the full Hamiltonian is broken by having $T_{k}^{0} \neq 0$; i.e., $\mathcal{T}^{\dagger} \mathcal{H}_{k} \mathcal{T} \neq \mathcal{H}_{-k}^{*}$, where $\mathcal{H}_{k}$ is the Hamiltonian density of Eq. (6) and $\mathcal{T}=\operatorname{diag}\left(\mathcal{T}_{1 \mathrm{D}}, \mathcal{T}_{\mathrm{sc}}\right)$.

We now project our system to an effective 1D model by integrating out the superconductor [47,51-57]. The superconductor induces a self-energy on the 1D system given by

$$
\Sigma_{k, \omega}=\int d \mathbf{r}_{\perp} \int d \mathbf{r}_{\perp}^{\prime} T_{k}^{\dagger}\left(\mathbf{r}_{\perp}\right) G_{k, \omega}^{\mathrm{sc}}\left(\mathbf{r}_{\perp}, \mathbf{r}_{\perp}^{\prime}\right) T_{k}\left(\mathbf{r}_{\perp}^{\prime}\right),
$$

where $G_{k, \omega}^{\mathrm{sc}}\left(\mathbf{r}_{\perp}, \mathbf{r}_{\perp}^{\prime}\right)$ is the Matsubara Green's function of the bare superconductor, defined such that $[i \omega-$ $\left.\mathcal{H}_{k}^{\mathrm{sc}}\left(\mathbf{r}_{\perp}\right)\right] G_{k, \omega}^{\mathrm{sc}}\left(\mathbf{r}_{\perp}, \mathbf{r}_{\perp}^{\prime}\right)=\delta\left(\mathbf{r}_{\perp}-\mathbf{r}_{\perp}^{\prime}\right)$. In the limit of weak tunneling, when the relevant pairing energies in the $1 \mathrm{D}$ system are $\omega \ll \Delta$, it is sufficient to evaluate the self-energy at $\omega=0$. In this case, the system is described by an effective 1D Hamiltonian given by $\mathcal{H}_{k}^{\text {eff }}=\mathcal{H}_{k}^{1 \mathrm{D}}+\Sigma_{k}$. Since it was already assumed that $\mathcal{H}_{k}^{1 \mathrm{D}}$ obeys an effective time-reversal symmetry, the Hamiltonian $\mathcal{H}_{k}^{\text {eff }}$ is in class DIII if the self-energy of Eq. (7) preserves this symmetry,

$$
\mathcal{T}_{1 \mathrm{D}}^{\dagger} \Sigma_{k} \mathcal{T}_{1 \mathrm{D}}=\Sigma_{-k}^{*}
$$

Hence, $\mathcal{T}_{1 \mathrm{D}}$ acts as an emergent time-reversal symmetry which exists only in the low-dimensional subspace. Assuming that the self-energy satisfies Eq. (8), we can decompose it into normal and anomalous parts as

$$
\Sigma_{k}=\tau_{z} \Sigma_{k}^{N}+\tau_{x} \Sigma_{k}^{A}
$$

where $\Sigma_{k}^{N}=G_{k}^{N}\left(T_{k}^{0 \dagger} T_{k}^{0}+T_{k}^{z \dagger} T_{k}^{z}\right)$ and $\Sigma_{k}^{A}=G_{k}^{A}\left(T_{k}^{0 \dagger} T_{k}^{0}-\right.$ $T_{k}^{z \dagger} T_{k}^{z}$ ) [60]. In arriving at Eq. (9), we have utilized the fact that the superconducting Green's function can be similarly decomposed as $G_{k}^{\mathrm{sc}}=\tau_{z} G_{k}^{N}+\tau_{x} G_{k}^{A}$, where $G_{k}^{N}$ and $G_{k}^{A}$ are scalars.

The anomalous self-energy, which represents the induced pairing in the $1 \mathrm{D}$ system, can be expressed in a form

$$
\Sigma_{k}^{A}=\Sigma_{k}^{z}-\Sigma_{k}^{0}
$$

where $\Sigma_{k}^{i}=-G_{k}^{A} T_{k}^{i \dagger} T_{k}^{i}$. It has been well demonstrated that the class DIII topological invariant can take a nontrivial value only if there exist at least two bands with opposite sign of the pairing potential $[38,40,41,50,61]$, or, equivalently, that the anomalous component of the self-energy has both positive and negative eigenvalues. It was also shown in Ref. [50] that $\Sigma_{k}^{z}$ is always a positive semidefinite matrix, and by extension it is straightforward to show that $\Sigma_{k}^{0}$ is also always positive semidefinite. Consequently, it is only possible for the DIII topological invariant to take a nontrivial value if both $\Sigma_{k}^{z}$ and $\Sigma_{k}^{0}$ are nonzero, as the anomalous self-energy is not restricted to be either positive or negative semidefinite in this case. Note that it was concluded in Ref. [50] that the DIII invariant always takes a trivial value because it was assumed that the full tunneling Hamiltonian [Eq. (6)] is time-reversal symmetric, and hence $T_{k}^{0}=\Sigma_{k}^{0}=0$. However, this is an unnecessarily restrictive assumption which is valid only for a certain subclass of one-dimensional DIII systems.

We have thus established three minimal criteria to realize a class DIII topological phase in a noninteracting 1D system: the bare $1 \mathrm{D}$ system must obey an effective time-reversal symmetry, the self-energy induced by the superconductor must preserve this symmetry, and the anomalous self-energy must have both positive and negative eigenvalues. The final requirement can be satisfied only if the full tunneling Hamiltonian [Eq. (6)] is not time-reversal invariant. We will now provide a model which satisfies all three criteria, showing indeed that the class DIII topological phase can be realized.

Model. We consider the geometry shown in Fig. 1. Two Rashba nanowires, separated by a distance $d$ (let us take one wire to be located at $z=0$ and the other at $z=d$ ), are coupled to an infinite 2D $s$-wave superconducting plane. We take the two nanowires to have opposite Zeeman splitting, which can be achieved by applying an antiparallel external magnetic field to each wire or by applying a uniform external magnetic field to two wires with opposite $g$-factors. 


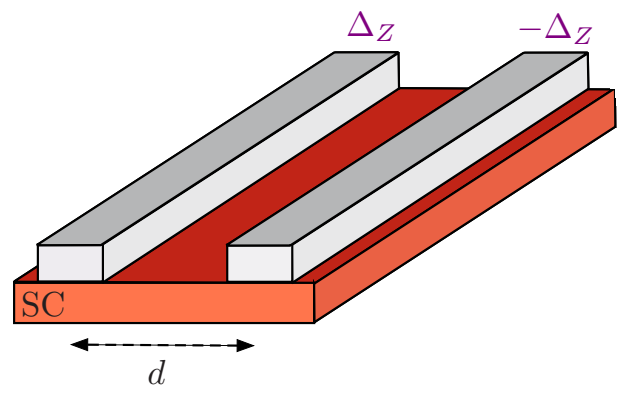

FIG. 1. Two nanowires, separated by a distance $d$, are coupled to an infinite superconducting plane. The two nanowires are assumed to have opposite Zeeman splittings.

The two nanowires are described by the Hamiltonian density

$$
h_{k}=\xi_{k}-\alpha k \sigma_{z}-\Delta_{Z} \lambda_{z} \sigma_{x},
$$

where $\xi_{k}=k^{2} / 2 m_{w}-\mu_{w}\left(m_{w}\right.$ is the effective mass and $\mu_{w}$ the chemical potential of the nanowires), $\alpha$ is the Rashba spin-orbit interaction constant (we choose our spin quantization axis along the direction of the effective Rashba field), and $\Delta_{Z}=g \mu_{B} B / 2$ is the Zeeman splitting in an external magnetic field of strength $B$ ( $g$ is the nanowire $g$-factor and $\mu_{B}$ is the Bohr magneton). The Pauli matrix $\lambda_{x, y, z}$ acts in left/right wire space. Crucially, we impose that the two nanowires are identical, with only a change in the sign of the Zeeman splitting. Although the Zeeman term in Eq. (11) explicitly breaks time reversal, the Hamiltonian density obeys an effective time-reversal symmetry $\mathcal{T}_{1 \mathrm{D}}^{\dagger} h_{k} \mathcal{T}_{1 \mathrm{D}}=h_{-k}^{*}$, where $\mathcal{T}_{1 \mathrm{D}}=i \lambda_{x} \sigma_{y}$ and $\mathcal{T}_{1 \mathrm{D}}^{2}=-1$.

The self-energy induced on the two nanowires by the superconductor is given in Eq. (7). Assuming that $\mu_{s} \gg \mu_{w}$, we evaluate the Green's function of the bulk 2D superconductor for momenta $k \ll k_{F s}\left(k_{F s}=\sqrt{2 m_{s} \mu_{s}}\right.$ is the Fermi momentum of the superconductor) to give

$$
\begin{aligned}
G_{0,0}^{\mathrm{sc}}\left(z, z^{\prime}\right)= & -\frac{1}{v_{F s}}\left[\tau_{x} \cos \left(k_{F s}\left|z-z^{\prime}\right|\right)\right. \\
& \left.-\tau_{z} \sin \left(k_{F s}\left|z-z^{\prime}\right|\right)\right] e^{-\left|z-z^{\prime}\right| / \xi_{s}},
\end{aligned}
$$

where $\xi_{s}=v_{F s} / \Delta$ is the coherence length and $v_{F s}=k_{F s} / m_{s}$ the Fermi velocity of the superconductor (we have also expanded in the limit $\mu_{s} \gg \Delta$ ). We assume local spinand momentum-independent tunneling of the form $t_{k}(z)=$ $\left[\begin{array}{ll}t \delta(z) & t \delta(z-d)\end{array}\right]$, where $t$ is a (scalar) tunneling amplitude which has the same strength in both nanowires. This gives $T_{k}^{0}=t_{k}\left(1-\lambda_{x}\right) / 2$ and $T_{k}^{z}=t_{k}\left(1+\lambda_{x}\right) / 2$. Evaluating the self-energy Eq. (7), we find

$$
\Sigma=\Gamma \tau_{z} \lambda_{x}+\tau_{x}\left(\Delta_{c}+\Delta_{d} \lambda_{x}\right),
$$

where we define a single-particle interwire tunnel coupling $\Gamma=\gamma \sin \left(k_{F s} d\right) e^{-d / \xi_{s}}$, an induced direct (intrawire) pairing potential $\Delta_{d}=\gamma$, and an induced crossed Andreev (interwire) pairing potential $\Delta_{c}=\gamma \cos \left(k_{F s} d\right) e^{-d / \xi_{s}}$ [47]. Here, $\gamma=$ $t^{2} / v_{F s}$ is a tunneling energy scale. Note that the pairing potentials always satisfy $\Delta_{d}>\Delta_{c}$.

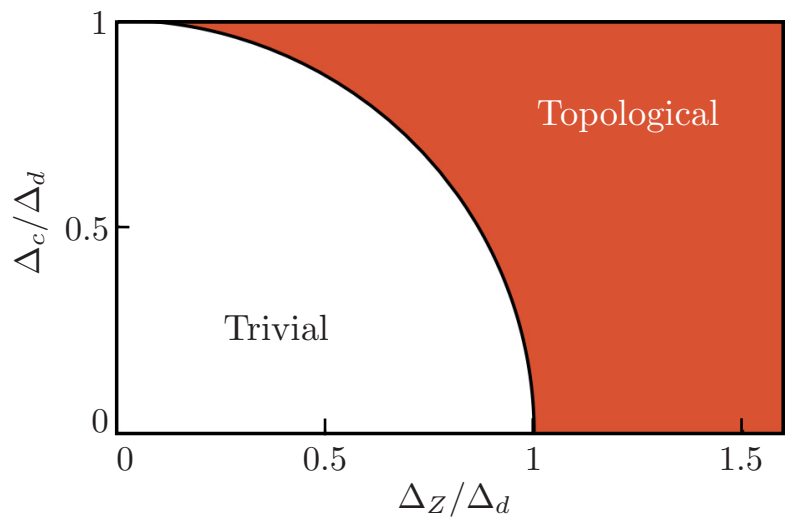

FIG. 2. Phase diagram of our model. A phase boundary at $\Delta_{Z}^{2}=$ $\Delta_{d}^{2}-\Delta_{c}^{2}$ separates trivial and DIII topological superconducting phases. The topological phase is characterized by the presence of a Kramers pair of Majorana fermions that is protected by the emergent time-reversal symmetry.

Taking into account the self-energy, the effective Hamiltonian describing the double nanowire system is given by

$\mathcal{H}_{k}^{\text {eff }}=\tau_{z}\left(\xi_{k}-\alpha k \sigma_{z}-\Delta_{Z} \lambda_{z} \sigma_{x}+\Gamma \lambda_{x}\right)+\tau_{x}\left(\Delta_{c}+\Delta_{d} \lambda_{x}\right)$.

Because the self-energy preserves the effective time-reversal symmetry, $\mathcal{T}_{1 \mathrm{D}}^{\dagger} \Sigma \mathcal{T}_{1 \mathrm{D}}=\Sigma^{*}$, we also have $\mathcal{T}_{1 \mathrm{D}}^{\dagger} \mathcal{H}_{k}^{\text {eff }} \mathcal{T}_{1 \mathrm{D}}=\mathcal{H}_{-k}^{\text {eff* }}$. Additionally, the effective Hamiltonian possesses a particlehole symmetry $\mathcal{P}^{\dagger} \mathcal{H}_{k}^{\text {eff }} \mathcal{P}=-\mathcal{H}_{-k}^{\text {eff* } *}$, where $\mathcal{P}=\tau_{y} \lambda_{x} \sigma_{y}$ is a unitary matrix satisfying $\mathcal{P}^{2}=1$. Finally, the effective Hamiltonian possesses a chiral symmetry $\left\{\mathcal{C}, \mathcal{H}_{k}^{\text {eff }}\right\}=0$, where $\mathcal{C}=\mathcal{T}_{1 \mathrm{D}} \mathcal{P}=i \tau_{y}$. These three properties of the Hamiltonian place it in the DIII symmetry class. Additionally, the anomalous self-energy $\Sigma^{A}=\Delta_{c}+\Delta_{d} \lambda_{x}$ has both positive and negative eigenvalues, $\Delta_{c} \pm \Delta_{d}$. Because all of our previously established criteria are met in this setup, it is possible to have a topological phase.

To determine whether such a topological phase exists in this setup, we search for a $k=0$ gap-closing transition by enforcing $\operatorname{det}\left(\mathcal{H}_{0}^{\text {eff }}\right)=0$. We find

$$
\begin{aligned}
\operatorname{det}\left(\mathcal{H}_{0}^{\text {eff }}\right)= & \left\{\Delta_{Z}^{4}+2 \Delta_{Z}^{2}\left(\Gamma^{2}-\Delta_{d}^{2}+\Delta_{c}^{2}-\mu_{w}^{2}\right)\right. \\
& +\left[\left(\Delta_{d}-\Delta_{c}\right)^{2}+\left(\Gamma-\mu_{w}\right)^{2}\right]\left[\left(\Delta_{d}+\Delta_{c}\right)^{2}\right. \\
& \left.\left.+\left(\Gamma+\mu_{w}\right)^{2}\right]\right\}^{2},
\end{aligned}
$$

which yields a gap closing at the critical Zeeman splitting $\left(\Delta_{Z}^{c}\right)^{2}=\Delta_{d}^{2}-\Delta_{c}^{2}+\mu_{w}^{2}-\Gamma^{2} \pm 2 i\left(\mu_{w} \Delta_{c}-\Gamma \Delta_{d}\right)$. Therefore, in order to have a physical transition, the chemical potential of the nanowires must be tuned to $\mu_{w}=\Gamma \Delta_{d} / \Delta_{c}$. For simplicity, let us assume that the system is tuned in such a way that the interwire tunnel coupling vanishes, $\Gamma=0$ [which can be done by tuning $\sin \left(k_{F s} d\right)=0$ ]. In this case, the critical Zeeman splitting at which the gap closes is given by

$$
\Delta_{Z}^{c}=\sqrt{\Delta_{d}^{2}-\Delta_{c}^{2}} \text {. }
$$

The phase diagram of our model is displayed in Fig. 2. We find two distinct phases whose topological characterization can be 
inferred along the line $\Delta_{c}=0$, corresponding to the case when the two wires are decoupled $\left(d \gg \xi_{s}\right)$. For $\Delta_{Z}<\Delta_{d}$, both wires are in a topologically trivial phase. For $\Delta_{Z}>\Delta_{d}$, both wires are in a topologically nontrivial phase, with each wire hosting its own distinct pair of Majorana bound states. Because the number of Majorana bound states is a topological invariant that cannot be changed without closing the gap, we conclude that $\Delta_{Z}^{2}<\Delta_{d}^{2}-\Delta_{c}^{2}$ corresponds to a topologically trivial phase while $\Delta_{Z}^{2}>\Delta_{d}^{2}-\Delta_{c}^{2}$ corresponds to a topologically nontrivial phase with two pairs of Majorana bound states.

To further establish the presence of a topologically nontrivial phase, we explicitly solve for the wave functions of the Majorana bound states. We now take our nanowires to be semi-infinite $(x>0)$, and we assume that the effective Hamiltonian of Eq. (14) remains valid for the semi-infinite case after replacing $k \rightarrow-i \partial_{x}$ [62]. States in the nanowires obey a Bogoliubov-de Gennes equation given by $\mathcal{H}_{\mathrm{eff}}(x) \phi(x)=$ $E \phi(x)$. By constructing a general zero-energy solution and imposing a vanishing boundary condition $\phi(0)=0$, we find two Majorana bound state solutions in the topological phase $\left(\Delta_{Z}^{2}>\Delta_{d}^{2}-\Delta_{c}^{2}\right)$ and no solutions in the trivial phase $\left(\Delta_{Z}^{2}<\right.$ $\left.\Delta_{d}^{2}-\Delta_{c}^{2}\right)$. The full analytical expressions for the wave functions are given in the limit of strong spin-orbit interaction $\left(m_{w} \alpha^{2} \gg \Delta_{d}, \Delta_{c}, \Delta_{Z}\right)$ in the Supplemental Material [60]. We find that the two Majorana wave functions in the topological phase are orthogonal,

$$
\phi_{2}^{\dagger}(x) \phi_{1}(x)=0,
$$

and related by the effective time-reversal symmetry,

$$
\begin{aligned}
& \phi_{1}(x)=\mathcal{T}_{1 \mathrm{D}} \phi_{2}^{*}(x), \\
& \phi_{2}(x)=-\mathcal{T}_{1 \mathrm{D}} \phi_{1}^{*}(x) .
\end{aligned}
$$

Hence, the two Majorana bound states in the system form a Kramers pair that is protected by the emergent time-reversal symmetry. We note that the Kramers degeneracy is lifted when the effective time-reversal symmetry is broken (for example by differences in the effective Hamiltonians of the two nanowires), which causes the Majorana bound states to split to finite energy. However, these subgap bound states persist at low energies provided that the symmetry breaking effect is smaller than the gap.

Conclusions. We have shown that a topological superconductor in symmetry class DIII can be realized in a noninteracting 1D system proximity coupled to a conventional superconductor. Crucially, the full Hamiltonian (incorporating the $1 \mathrm{D}$ system, the parent superconductor, and the tunneling term) must not possess an effective time-reversal symmetry, with such a symmetry emerging only after projection to an effective 1D model. We provide an explicit example realizing such a class DIII topological superconductor, showing that the topological phase is characterized by a Kramers pair of Majorana bound states which is protected by the effective time-reversal symmetry of the system. We believe that our general criteria can be applied to realize class DIII topological superconductivity in a multitude of additional systems coupled to a bulk $s$-wave superconductor, for example in nanowires with helical magnetization of opposite helicity, antiferromagnetically coupled spin chains, magnetic topological insulators with opposite magnetization, or ferromagnetic atomic chains with opposite magnetization.

Note added. Upon completion of this manuscript, we became aware of a very recent preprint, Ref. [63], which discusses the realization of a class DIII topological phase in 2D antiferromagnetic quantum spin Hall insulators, providing an additional physical realization of the three topological criteria proposed in the present Rapid Communication.

Acknowledgments. This work was supported by the Swiss National Science Foundation and the NCCR QSIT.
[1] J. Alicea, Rep. Prog. Phys. 75, 076501 (2012).

[2] M. Leijnse and K. Flensberg, Semicond. Sci. Technol. 27, 124003 (2012).

[3] C. W. J. Beenakker, Annu. Rev. Condens. Matter Phys. 4, 113 (2013).

[4] A. Y. Kitaev, Phys. Usp. 44, 131 (2001).

[5] C. Nayak, S. H. Simon, A. Stern, M. Freedman, and S. Das Sarma, Rev. Mod. Phys. 80, 1083 (2008).

[6] R. M. Lutchyn, J. D. Sau, and S. Das Sarma, Phys. Rev. Lett. 105, 077001 (2010).

[7] Y. Oreg, G. Refael, and F. von Oppen, Phys. Rev. Lett. 105, 177002 (2010).

[8] J. Alicea, Phys. Rev. B 81, 125318 (2010).

[9] D. Chevallier, D. Sticlet, P. Simon, and C. Bena, Phys. Rev. B 85, 235307 (2012).

[10] B. I. Halperin, Y. Oreg, A. Stern, G. Refael, J. Alicea, and F. von Oppen, Phys. Rev. B 85, 144501 (2012).

[11] D. Sticlet, C. Bena, and P. Simon, Phys. Rev. Lett. 108, 096802 (2012).

[12] J. Klinovaja, P. Stano, and D. Loss, Phys. Rev. Lett. 109, 236801 (2012).
[13] E. Prada, P. San-Jose, and R. Aguado, Phys. Rev. B 86, 180503(R) (2012).

[14] F. Domínguez, F. Hassler, and G. Platero, Phys. Rev. B 86, 140503 (2012).

[15] W. DeGottardi, M. Thakurathi, S. Vishveshwara, and D. Sen, Phys. Rev. B 88, 165111 (2013).

[16] E. Vernek, P. H. Penteado, A. C. Seridonio, and J. C. Egues, Phys. Rev. B 89, 165314 (2014).

[17] V. Mourik, K. Zuo, S. M. Frolov, S. R. Plissard, E. P. A. M. Bakkers, and L. P. Kouwenhoven, Science 336, 1003 (2012).

[18] M. T. Deng, C. L. Yu, G. Y. Huang, M. Larsson, P. Caroff, and H. Q. Xu, Nano Lett. 12, 6414 (2012).

[19] A. Das, Y. Ronen, Y. Most, Y. Oreg, M. Heiblum, and H. Shtrikman, Nat. Phys. 8, 887 (2012).

[20] L. P. Rokhinson, X. Liu, and J. K. Furdyna, Nat. Phys. 8, 795 (2012).

[21] H. O. H. Churchill, V. Fatemi, K. Grove-Rasmussen, M. T. Deng, P. Caroff, H. Q. Xu, and C. M. Marcus, Phys. Rev. B 87, 241401 (2013).

[22] A. D. K. Finck, D. J. Van Harlingen, P. K. Mohseni, K. Jung, and X. Li, Phys. Rev. Lett. 110, 126406 (2013). 
[23] W. Chang, S. M. Albrecht, T. S. Jespersen, F. Kuemmeth, P. Krogstrup, J. Nygård, and C. M. Marcus, Nat. Nanotechnol. 10, 232 (2015).

[24] S. M. Albrecht, A. P. Higginbotham, M. Madsen, F. Kuemmeth, T. S. Jespersen, J. Nygård, P. Krogstrup, and C. M. Marcus, Nature (London) 531, 206 (2016).

[25] M. T. Deng, S. Vaitiekenas, E. B. Hansen, J. Danon, M. Leijnse, K. Flensberg, J. Nygård, P. Krogstrup, and C. M. Marcus, Science 354, 1557 (2016).

[26] H. Zhang, Ö. Gül, S. Conesa-Boj, M. Nowak, M. Wimmer, K. Zuo, V. Mourik, F. K. de Vries, J. van Veen, M. W. A. de Moor, J. D. S. Bommer, D. J. van Woerkom, D. Car, S. R. Plissard, E. P. A. M. Bakkers, M. Quintero-Pérez, M. C. Cassidy, S. Koelling, S. Goswami, K. Watanabe, T. Taniguchi, and L. P. Kouwenhoven, Nat. Commun. 8, 16025 (2017).

[27] S. Nadj-Perge, I. K. Drozdov, B. A. Bernevig, and A. Yazdani, Phys. Rev. B 88, 020407 (2013).

[28] F. Pientka, L. I. Glazman, and F. von Oppen, Phys. Rev. B 88, 155420 (2013).

[29] J. Klinovaja, P. Stano, A. Yazdani, and D. Loss, Phys. Rev. Lett. 111, 186805 (2013)

[30] M. M. Vazifeh and M. Franz, Phys. Rev. Lett. 111, 206802 (2013).

[31] B. Braunecker and P. Simon, Phys. Rev. Lett. 111, 147202 (2013).

[32] S. Nadj-Perge, I. K. Drozdov, J. Li, H. Chen, S. Jeon, J. Seo, A. H. MacDonald, B. A. Bernevig, and A. Yazdani, Science 346, 602 (2014).

[33] Y. Peng, F. Pientka, L. I. Glazman, and F. von Oppen, Phys. Rev. Lett. 114, 106801 (2015).

[34] R. Pawlak, M. Kisiel, J. Klinovaja, T. Meier, S. Kawai, T. Glatzel, D. Loss, and E. Meyer, Npj Quant. Info. 2, 16035 (2016).

[35] A. P. Schnyder, S. Ryu, A. Furusaki, and A. W. W. Ludwig, Phys. Rev. B 78, 195125 (2008).

[36] C. L. M. Wong and K. T. Law, Phys. Rev. B 86, 184516 (2012).

[37] S. Nakosai, J. C. Budich, Y. Tanaka, B. Trauzettel, and N. Nagaosa, Phys. Rev. Lett. 110, 117002 (2013).

[38] F. Zhang, C. L. Kane, and E. J. Mele, Phys. Rev. Lett. 111, 056402 (2013).

[39] E. Dumitrescu, J. D. Sau, and S. Tewari, Phys. Rev. B 90, 245438 (2014).

[40] A. Keselman, L. Fu, A. Stern, and E. Berg, Phys. Rev. Lett. 111, 116402 (2013).

[41] A. Haim, A. Keselman, E. Berg, and Y. Oreg, Phys. Rev. B 89, 220504 (2014).
[42] C. Schrade, A. A. Zyuzin, J. Klinovaja, and D. Loss, Phys. Rev. Lett. 115, 237001 (2015).

[43] J. Klinovaja and D. Loss, Phys. Rev. B 90, 045118 (2014).

[44] E. Gaidamauskas, J. Paaske, and K. Flensberg, Phys. Rev. Lett. 112, 126402 (2014).

[45] C. Schrade, M. Thakurathi, C. Reeg, S. Hoffman, J. Klinovaja, and D. Loss, Phys. Rev. B 96, 035306 (2017)

[46] J. Klinovaja, A. Yacoby, and D. Loss, Phys. Rev. B 90, 155447 (2014).

[47] C. Reeg, J. Klinovaja, and D. Loss, Phys. Rev. B 96, 081301 (2017).

[48] P. Recher and D. Loss, Phys. Rev. B 65, 165327 (2002).

[49] C. Bena, S. Vishveshwara, L. Balents, and M. P. A. Fisher, Phys. Rev. Lett. 89, 037901 (2002).

[50] A. Haim, E. Berg, K. Flensberg, and Y. Oreg, Phys. Rev. B 94, 161110 (2016).

[51] J. D. Sau, R. M. Lutchyn, S. Tewari, and S. Das Sarma, Phys. Rev. B 82, 094522 (2010).

[52] A. C. Potter and P. A. Lee, Phys. Rev. B 83, 184520 (2011).

[53] N. B. Kopnin and A. S. Melnikov, Phys. Rev. B 84, 064524 (2011).

[54] A. A. Zyuzin, D. Rainis, J. Klinovaja, and D. Loss, Phys. Rev. Lett. 111, 056802 (2013).

[55] B. van Heck, R. M. Lutchyn, and L. I. Glazman, Phys. Rev. B 93, 235431 (2016).

[56] C. Reeg and D. L. Maslov, Phys. Rev. B 95, 205439 (2017).

[57] C. Reeg, D. Loss, and J. Klinovaja, Phys. Rev. B 96, 125426 (2017).

[58] S. Tewari and J. D. Sau, Phys. Rev. Lett. 109, 150408 (2012).

[59] Note that we do not consider the possibility that $t_{k}$ is a two-body operator as in Ref. [42], as this corresponds to an interacting model.

[60] See Supplemental Material at http://link.aps.org/supplemental/ 10.1103/PhysRevB.96.161407 for a derivation of Eq. (9) and a detailed solution for the Majorana wave functions of our model.

[61] X.-L. Qi, T. L. Hughes, and S.-C. Zhang, Phys. Rev. B 81, 134508 (2010).

[62] Because the proximity effect was treated in the bulk of the nanowires, there could be additional boundary effects that are not properly accounted for in the effective Hamiltonian. However, the presence or absence of topological Majorana modes is insensitive to such boundary effects.

[63] Y. Huang and C.-K. Chiu, arXiv:1708.05724. 